REVISTA DE GEOCIÊNCIAS DO NORDESTE

Northeast Geosciences Journal

v. $6, n^{\circ} 2(2020)$

ISSN: $2447-3359$

https://doi.org/10.21680/2447-3359.2020v6n2ID19029

\title{
ESTUDO DA COMPOSIÇÃO E ABUNDÂNCIA DE ARANHAS (ARACHNIDA: ARANEAE) DE SOLO EM DUAS FITOFISIONOMIAS DO CERRADO, MARANHÃO, BRASIL
}

\author{
Francisco Ideilson Lima Soares ${ }^{1}$; Luciana Alves \\ da Luz Soares²; Luana da Luz dos Reis ${ }^{3}$; Alana \\ Ellen de Sousa Martins ${ }^{4}$; Judson Chaves \\ Rodrigues $^{5}$; Antonio Domingos Brescovit'; Luiza \\ Daiana Araújo da Silva Formiga ${ }^{7}$
}

${ }^{1}$ Graduado em Ciências Biológicas, Departamento de Química e Biologia, Universidade Estadual do Maranhão (UEMA), Caxias/MA, Brasil.

ORCID: https://orcid.org/0000-0002-6655-9048

Email: idesoares_lima@hotmail.com

${ }^{2}$ Doutora em Biologia Ambiental, Instituto de Estudos Costeiros, Universidade Federal do Pará (UFPA), Bragança/PA, Brasil.

ORCID: https://orcid.org/0000-0002-6756-8034

Email: lucianaluz_alves@hotmail.com

${ }^{3}$ Doutora em Biologia Ambiental, Instituto de Estudos Costeiros, Universidade Federal do Pará (UFPA), Bragança/PA, Brasil.

ORCID: https://orcid.org/0000-0002-6226-5077

Email: luana_luzreis@hotmail.com

${ }^{4}$ Graduanda em Ciências Biológicas, Departamento de Química e Biologia, Universidade Estadual do Maranhão (UEMA), Caxias/MA, Brasil.

ORCID: https://orcid.org/0000-0002-3543-8972

Email: a.lanasousa2009@hotmail.com

${ }^{5}$ Graduando em Ciências Biológicas, Departamento de Química e Biologia, Universidade Estadual do Maranhão (UEMA), Caxias/MA, Brasil.

ORCID: https://orcid.org/0000-0001-9236-2508

Email: judsoom.rodriguesz@gmail.com

${ }^{6}$ Doutor em Zoologia, Instituto Butantan, São Paulo/SP, Brasil. ORCID: https://orcid.org/0000-0002-1511-5324

Email: antonio.brescovit@butantan.gov.br
${ }^{7}$ Doutora em Zootecnia, Universidade Federal da Paraíba (UFPB), João Pessoa/PB, Brasil.

ORCID: https://orcid.org/0000-0001-5001-3297

Email: luizadaiana@hotmail.com

\section{Resumo}

O Brasil é um dos países com a maior diversidade de aranhas do mundo. No entanto, as estimativas em relação a real diversidade desses animais são bastante incipientes no Nordeste, principalmente no Maranhão. Foram coletados um total de 543 espécimes de aranhas de solo em duas fitofisionomias de Cerrado (Mata de Galeria e Cerrado sensu stricto) localizadas na APA Municipal do Inhamun, Caxias, Maranhão. Foram identificadas 23 famílias, 37 gêneros e 43 espécies (infraordem Araneomorphae), sendo que a maioria das espécies encontradas são registradas pela primeira vez para o estado do Maranhão. As famílias Corinnidae e Oonopidae apresentaram maior riqueza de espécies, enquanto a família Zodariidae a maior abundância de indivíduos. As espécies mais abundantes neste estudo foram Leprolochus birabeni e Corinna sp.1.

Palavras-chave: Araneofauna; Edáfica; Diversidade.

STUDY OF COMPOSITION AND ABUNDANCE OF GROUND SPIDERS (ARACHNIDA: ARANEAE) IN TWO PHYTOPHYSIOGNOMIES OF THE CERRADO BIOME IN MARANHÃO, BRAZIL

\section{Abstract}

Brazil is one of the countries with the largest diversity of spiders in the world. However, estimates regarding the real diversity of these animals are incipient in the Northeast, especially in the state of Maranhão. A total of 543 specimens of ground spiders were collected in two phytophysiognomies of the cerrado biome (gallery forest and cerrado sensu stricto) located in the Municipal APA of Inhamun, Caxias, Maranhão. We identified 23 families, 37 genera and 43 species (Araneomorphae infraorder), and most of the species found are recorded being for the first time for the state of Maranhão. The families Corinnidae and Oonopidae exhibited higher species richness, whereas the family Zodariidae 
showed the largest abundance of individuals. The most abundant species in this study were Leprolochus birabeni and Corinna sp.1.

Keywords: Spider fauna; Edaphic; Diversity.

ESTUDIO DE LA COMPOSICIÓN Y ABUNDANCIA DE ARAÑAS (ARACHNIDA: ARANEAE) DEL SUELO EM DOS FITOFISIOGNOMÍAS EM EL CERRADO, MARANHÃO, BRASIL

\section{Resumen}

Brasil es uno de los países con mayor diversidad de arañas del mundo. Sin embargo, las estimaciones con respecto a la diversidad real de estos animales son bastante incipientes en el Nordeste, especialmente en Maranhão. Se recolectaron un total de 543 especímenes de arañas del suelo en dos fitofisiognomías en el Cerrado (Mata de Galeria y Cerrado sensu stricto) ubicado en el Inhamun Municipal APA, Caxias, Maranhão. Se identificaron 23 familias, 37 géneros y 43 especies (infraorden Araneomorphae), y la mayoría de las especies se registraron por primera vez en el estado de Maranhão. Las familias Corinnidae y Oonopidae tuvieron la mayor riqueza de especies, mientras que la familia Zodariidae la mayor abundancia de individuos. Las especies más abundantes en este estudio fueron Leprolochus birabeni y Corinna sp.1.

Palabras-clave: Araneofauna; Edáfica; Diversidad.

\section{INTRODUÇÃO}

O Cerrado compreende o segundo maior bioma em área original do Brasil e sua heterogeneidade fisionômica revela uma diversidade ambiental acentuada (CONCEIÇÃO; CASTRO, 2009). No Nordeste do Brasil as maiores concentrações dos cerrados encontram-se nos estados do Piauí e do Maranhão, este, com aproximadamente 10.000.000 hectares, distribuídos em mais de 38 municípios (NERES; CONCEIÇÃO, 2010). Apesar de sua diversidade, a degradação do Cerrado tem sido intensiva (LEITE et al., 2010), em conseguinte, novos estudos sobre a dinâmica e a composição das espécies nesse bioma são incentivados (CARVALHO; AVELINO, 2010).

O Brasil é um dos países com a maior diversidade de aranhas do mundo (BRESCOVIT; OLIVEIRA; SANTOS, 2011). A ordem Araneae é o segundo maior grupo entre os aracnídeos, com aproximadamente 47.663 espécies descritas, distribuídas mundialmente em 117 famílias (World Spider Catalog, 2020). Para o Cerrado já foram registradas 625 espécies, das quais 169 são endêmicas deste bioma (OLIVEIRA; BRESCOVIT; SANTOS, 2017). A ordem Araneae está dividida em duas subordens: Mesothelae (aracnídeos com características primitivas) e Opisthothele (engloba todas as aranhas restantes), sendo esta representada por duas infraordens: Mygalomorphae e Araneomorphae (FOELIX, 2011).

As aranhas são reconhecidas como bioindicadores de qualidade ambiental, por sua extrema sensibilidade em resposta às perturbações naturais e antrópicas e são reconhecidas ainda, como agentes de controle biológico para insetos fitófagos (ROCHA, 2017). Apesar de sua importância ecológica o grupo representa o táxon menos estudado para o Cerrado, com poucos registros de enfoque taxonômico e de distribuição geográfica
(CARVALHO; AVELINO, 2010; MORAES, 2014; SANTANA, 2015; OLIVEIRA; BRESCOVIT; SANTOS, 2017).

Estudos sobre as aranhas de solo na APA do Inhamun encontram-se mal amostrados, sendo esta considerada como um importante patrimônio ecológico local. Diante disso, o presente estudo teve como objetivo realizar um levantamento da composição e abundância de aranhas de solo em duas fitofisionomias de Cerrado da Área de Proteção Ambiental Municipal do Inhamum (APA do Inhamum), em Caxias, no estado do Maranhão. Este estudo representa uma contribuição para o conhecimento da biodiversidade de aranhas na região sendo o primeiro levantamento padronizado realizado.

\section{METODOLOGIA}

\subsection{Caracterização da área de estudo}

As coletas foram realizadas em duas áreas experimentais delimitadas na Área de Proteção Ambiental Municipal do Inhamum - APA do Inhamun, no município de Caxias, Maranhão, representando duas fitofisionomias típicas do Cerrado: Mata de Galeria e Cerrado sensu stricto (Figura 1). A APA do Inhamum é caracterizada por apresentar clima subúmido seco, com dois períodos bem definidos, um chuvoso de janeiro a junho e outro seco de julho a dezembro (ALBUQUERQUE, 2012). Apresenta índices pluviométricos regulares entre 1.600 e $1.800 \mathrm{~mm}$, com temperaturas normalmente elevadas com média anual superior a $24^{\circ} \mathrm{C}$. A vegetação local é composta por gramíneas em área plana, característico de cerradões, chapadas, cerrado e pequenos pontos de mata fechada (ALBUQUERQUE, 2012; CONCEIÇÃO; da SILVA; RODRIGUES, 2014; da SILVA et al. 2016). No Bioma Cerrado, a Mata de Galeria é caracterizada por arbustos e árvores dispersas com grandes quantidades de gramíneas e serapilheira, com dossel aberto à semiaberto e fechado em alguns pontos. As famílias vegetais predominantes são: Fabaceae, Myrtaceae, Poaceae e Rubiaceae (da SILVA et al., 2016). O Cerrado sensu stricto apresenta uma vegetação típica, com árvores de pequeno a médio porte, arbustos, com dossel aberto à semiaberto e com destaque para as famílias Areacaceae, Fabaceae, Caryocaraceae, Rubiaceae, entre outras (CONCEIÇÃO; da SILVA; RODRIGUES, 2014).

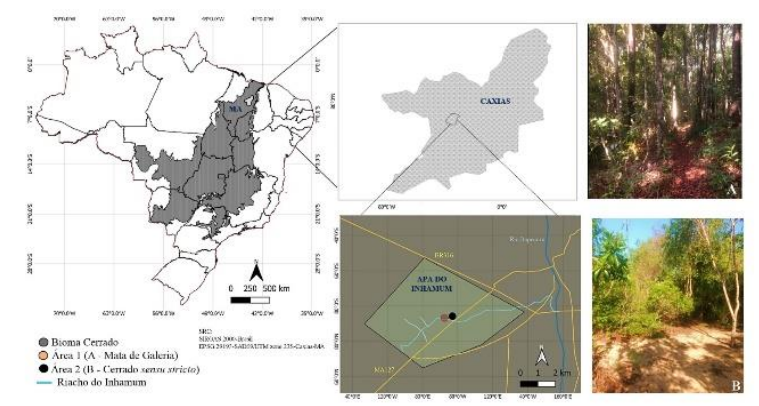

Figura 1 - Mapa da Área de Proteção Ambiental Municipal do Inhamum na Cidade de Caxias/MA, com destaque para as fitofisionomias estudadas. Fonte: elaborado pelos autores (2020). 


\subsection{Coleta e identificação do material}

A coleta do material foi realizada durante o período seco (outubro, novembro e dezembro de 2017) e período chuvoso (janeiro, fevereiro, março e abril de 2018), utilizando armadilhas do tipo Provid (FORNAZIER et al., 2007; GIRACCA et al., 2003). As armadilhas foram distribuídas na Mata de Galeria (Área 1) e Cerrado sensu stricto (Área 2) e em cada área foram estabelecidos seis transectos paralelos, com distância de aproximadamente $10 \mathrm{~m}$ entre si, enquanto cada transecto foi marcado por cinco unidades amostrais equidistantes $(10 \mathrm{~m} \mathrm{x} 10$ $\mathrm{m})$, de modo que foram amostrados 30 pontos em cada área, totalizando 60 pontos. As armadilhas permaneceram no campo por um período de 96 horas (DRESCHER et al., 2007).

Após esse período, o material foi retirado do solo e levado para o laboratório para triagem manual utilizando peneiras de malha de $0.25 \mathrm{~mm}$. As aranhas coletadas foram fixadas em álcool etílico a $70 \%$ e enviadas para a identificação no Instituto Butantan. Os indivíduos adultos foram identificados ao nível de espécies ou morfoespécies, enquanto os imaturos foram contabilizados e descartados, não entrando nas análises qualitativas. Todo o material está depositado no acervo do Laboratório de Coleções Zoológicas do Instituto Butantan (Curador: A. D. Brescovit). A coleta de espécimes foi autorizada pelo ICMBio/IBAMA, Instituto Federal do Meio Ambiente (Licença número 583781).

\subsection{Análises estatísticas}

O monitoramento da temperatura do solo $(10 \mathrm{~cm}$ de profundidade) foi realizado com auxílio de Termômetro Digital Tipo Espeto. Os dados de precipitação durante os períodos de coleta foram obtidos na Estação Meteorológica de Caxias, Maranhão. Para as análises estatísticas inicialmente foi produzido um banco de dados no programa Software Microsoft Excel, e a partir deste, foi construída uma tabela com a composição das aranhas encontradas nas duas áreas. As análises faunísticas com base nos índices de dominância, abundância, frequência e constância dos táxons coletados foram feitas no programa ANAFAU (MORAES et al., 2003). Também foram gerados gráficos para correlacionar os fatores físicos (temperatura e pluviosidade) com a riqueza e abundância dos indivíduos coletados.

\section{RESULTADOS E DISCUSSÃO}

Foram coletados um total de 543 espécimes de aranhas de solo, sendo 253 adultos (46.60\%) e 290 juvenis (53.40\%). Do total de espécimes adultos, 111 foram coletados na Área 1 (43.87\% - Mata de Galeria) e 142 indivíduos foram provenientes da Área 2 (56.13\% - Cerrado sensu stricto). Entre os adultos contabilizados, $71.15 \%$ eram machos (180 indivíduos) e $28.85 \%$ eram fêmeas (73 indivíduos). Neste levantamento foram identificadas 23 famílias, 37 gêneros e 43 espécies pertencentes à infraordem Araneomorphae (Tabela 1).

$\mathrm{Na}$ Área 1 foram registradas 31 espécies, distribuídas em 17 famílias, das quais quatro foram exclusivas para a Mata de Galeria: Caponiidae, Mysmenidae, Pisauridae e Theridiidae. As famílias, com maior riqueza de espécies foram Corinnidae e
Oonopidae. Em relação a abundância de indivíduos, duas famílias se mostraram expressivas: Zodariidae e Corinnidae, que juntas somaram $44 \%$ do total das aranhas de solo coletadas na Área 1 (Tabela 1).

$\mathrm{Na}$ Área 2 foram registradas 32 espécies, distribuídas em 19 famílias, das quais seis ocorreram apenas no Cerrado sensu stricto: Hahniidae, Miturgidae, Prodidomidae, Sicariidae, Scytodidae e Theraphosidae. As famílias Corinnidae e Oonopidae foram, as que apresentaram maior riqueza de espécies. No que se refere à abundância de indivíduos, as famílias Zodariidae e Corinnidae também foram as mais representativas, correspondendo a 53\% das aranhas de solo coletadas na Área 2 (Tabela 1).

A expressividade das famílias Corinnidae, Oonopidae e Zodariidae também foram relatadas em outros estudos sobre a araneofauna de solo em Biomas de Cerrado da região Nordeste (DIAS; BRESCOVIT; MENEZES, 2005; CARVALHO; AVELINO, 2010; CUNHA et al., 2012; AZEVEDO et al., 2017). Analisando a composição e riqueza de aranhas de solo em dois fragmentos de Cerrado Litorâneo no Maranhão, Cunha et al. (2012) observaram que as famílias mais abundantes foram Corinnidae e Zodariidae, sendo esta última bem representativa em ambos as áreas analisadas (Tutóia e Paulino Neves). Ainda segundo os autores, a família Corinnidae foi a que apresentou maior riqueza de espécies.

Carvalho e Avelino (2010) realizaram um levantamento de aranhas de solo em quatro fitofisionomias do Bioma Cerrado (mata dos cocais, mata seca semi-decídua primária, mata seca semi-decídua secundária e cerrado típico), em uma fazenda no Município de José de Freitas no Piauí. Neste estudo a família Corinnidae apresentou o maior número de espécies, enquanto Zodariidae apresentou maior abundância de indivíduos.

Ao comparar a abundância relativa e composição da araneofauna de solo em seis ambientes florestais (cacaual em cabruca, capoeira, borda e interior de fragmento de mata e borda e interior de mata contínua) nos municípios de Ilhéus e Una na Bahia, Dias, Brescovit e Menezes (2005) observaram que as famílias Oonopidae e Corinnidae se destacaram em relação a abundância de indivíduos e riqueza de espécies, respectivamente.

Dentre as 43 espécies identificadas, 18 foram singletons ou raras (r) e oito doubletons ou dispersas (d) (Tabela 1). Em ambas as áreas analisadas a espécie mais abundante foi Leprolochus birabeni Mello-Leitão 1942, com 66 indivíduos, sendo estes capturados em todos os meses de coleta. A maioria das espécies encontradas neste estudo é registrada pela primeira vez para o estado do Maranhão, representando $70 \%$ das espécies identificadas (Tabela 1).

O primeiro registro sobre a composição da araneofauna de solo para os Cerrados do estado do Maranhão, foi realizado por Cunha et al. (2012) em duas áreas litorâneas (Tutóia e Paulino Neves). Neste levantamento foi obtido um total de 234 aranhas, sendo 142 adultos (61\%) e 92 jovens (39\%), distribuídas em 12 famílias, 18 gêneros e 19 espécies. Assim como foi observado em nossos resultados, a família que também apresentou o maior número de indivíduos foi Zodariidae e a espécie mais abundante foi Leprolochus sp.

Três espécies foram dominantes (D) tanto na Área 1 como na Área 2: Corinna sp.1, Kambiwa sp. e L. birabeni. Foram ainda dominantes apenas na Mata de Galeria (Área 1), as espécies 
Ctenus sp.1 e Ochyrocera sp. e no Cerrado stricto sensu (Área 2), as espécies Camillina sp., Epicratinus sp. e Oonopoides sp.1. As demais espécies registradas foram classificadas como não dominantes (ND) com frequências inferiores entre $2-5 \%$.

As espécies, que se mostraram dominantes foram, consequentemente, as que também apresentaram maior frequência, sendo consideradas como muito frequentes (MF): Área 1 - Corinna sp.1, Ctenus sp.1, Kambiwa sp., L. birabeni, Ochyrocera sp., e na Área 2 - Camillina sp., Corinna sp.1, Epicratinus sp., Kambiwa sp., L. birabeni e Oonopoides sp.1. As espécies qualificadas como frequentes (F) foram: Área 1 - Ctenus sp.2, Hexapopha sp., Oonopoides sp.2, Otiothops sp., Parachemmis sp., Soesilarishius sp., Sphecozone sp. e na Área 2 - Ctenus sp.1, Goeldia sp., Hexapopha sp.1, Neotrops sp. e Neoxyphinus sp. As demais espécies encontradas obtiveram frequências menores, que o limite do intervalo de confiança de $5 \%$ e, portanto, foram classificadas como pouco frequentes (PF).

Com relação a constância das aranhas de solo coletadas, na Mata de Galeria (Área 1), quatro espécies foram consideradas como constantes (W) (Corinna sp., Kambiwa sp., L. birabeni e Ochyrocera sp.) e oito espécies como acessórias (Y) (Ctenus sp.1, Ctenus sp.2, Hexapopha sp.1, Oonopoides sp.2, Otiothops sp., Parachemmis sp., Soesilarishius sp., Sphecozone sp.). No Cerrado stricto sensu (Área 2), apenas a espécie L. birabeni foi considerada como constante, enquanto treze espécies foram classificadas como acessórias (Apopyllus sp., Camillina sp., Corinna sp.1, Ctenus sp.1, Epicratinus sp., Goeldia sp., Hexapopha sp.1, Kambiwa sp., Neotrops sp., Neoxyphinus sp., Oonopoides sp.1, Soesilarishius sp. e Teminius insularis). No entanto, a maioria das espécies registradas em ambas as áreas estiveram presentes em menos de $20 \%$ das coletas e, portanto, foram consideradas como acidentais $(\mathrm{Z})$.

As espécies Corinna sp. 1 e $L$. birabeni foram as mais dominantes, frequentes e com maior constância tanto na Mata de Galeria como no ambiente Cerrado stricto sensu. Outros estudos também têm relatado a dominância dessas espécies de aranhas em fitofisionomias de Cerrado no Maranhão e em outros estados do Nordeste (DIAS; BRESCOVIT; MENEZES, 2005; CARVALHO; AVELINO, 2010; CUNHA et al., 2012; AZEVEDO et al., 2017).

A variação na riqueza e abundância das aranhas de solo foi influenciada de formas diferenciadas pela temperatura do solo $\left({ }^{0} \mathrm{C}\right)$ e precipitação pluvial $(\mathrm{mm})$ durante os períodos de coleta (seco e chuvoso). Não foi encontrada diferença expressiva na riqueza total de espécies registradas entre o período seco (Área 1=25; Área 2=22) e chuvoso (Área 1=31; Área 2=33). Na Mata de Galeria foi observada maior riqueza de espécies entre os meses de dezembro $(n=11)$ e janeiro $(n=10)$, durante a passagem da estação seca para a chuvosa. No Cerrado sensu stricto o maior registro no número de espécies foi observado no período chuvoso entres os meses de janeiro $(n=8)$, fevereiro $(n=11)$ e março $(n=$ 9) (Figura 2).

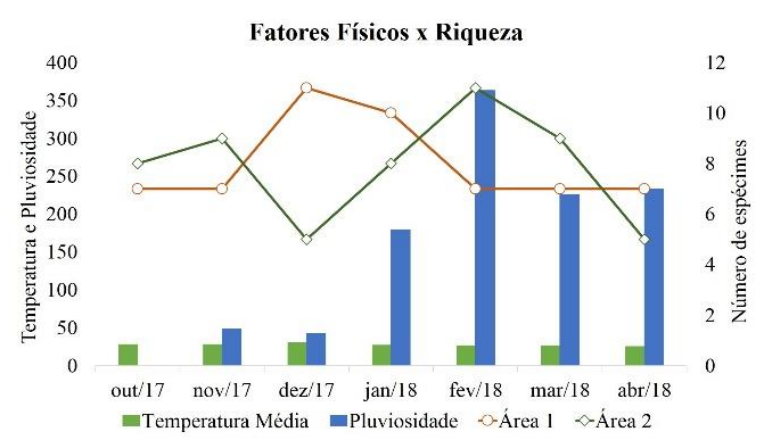

Figura 2 - Variação da riqueza de espécies em função da temperatura do solo e pluviosidade na APA do Inhamun, Caxias - MA, no período de outubro/2017 a abril/2018. Fonte: elaborado pelos autores (2019).

Em termos de abundância de indivíduos, o número de espécimes coletados no período seco foi praticamente constante nas duas áreas (Área 1= 59; Área 2=57), embora para a Mata de Galeria, o maior registro de indivíduos tenha sido no mês de dezembro/2017 e para o Cerrado sensu stricto no mês de outubro/2017. Entretanto, na estação chuvosa houve um decréscimo na abundância de espécimes coletados na Área 1 ( $n=$ 52) em comparação com a Área $2(n=85)$, principalmente nos primeiros meses amostrados (Figura 3 ).

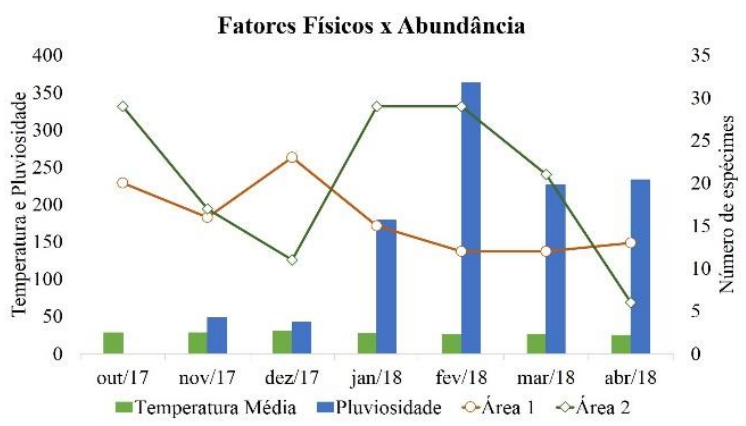

Figura 3 - Variação na abundância de espécimes em função da temperatura do solo e pluviosidade na APA do Inhamun, Caxias - MA, no período de outubro/2017 a abril/2018. Fonte: elaborado pelos autores (2019).

A maior abundância de aranhas nos meses mais quentes se deve ao fato destas estarem mais ativas na procura de presas e para se reproduzir, principalmente os machos, enquanto nos meses mais frios há um decréscimo na abundância, já que a disponibilidade de presas diminui consideravelmente, fazendo com que as mesmas tenham que estocar energia e não se encontram forrageando com a mesma frequência (FERRO, 2008; FRANCISCO, 2011; SOUSA, 2016; LIMA et al., 2019).

Na passagem da estação seca para chuvosa, foi observado um aumento no número de indivíduos registrados no Cerrado sensu stricto $(\mathrm{n}=85)$, especialmente nos meses de janeiro e fevereiro/2018, correspondendo a $60 \%$ dos indivíduos coletados na Área 2 durante esse período, embora esse índice tenha diminuído gradativamente nos meses seguintes. Na Mata de Galeria, embora a passagem da estação seca para chuvosa tenha 
sido marcada pela diminuição no registro de indivíduos, nos meses de fevereiro, março e abril/2018, esse número se manteve constante ( $\mathrm{n}=12,12$ e 13, respectivamente) (Figura 3).

Curiosamente, as duas espécies mais abundantes (L. birabeni e Corinna sp.1) mostraram variações no número de indivíduos registrados durante o período seco e chuvoso. A espécie $L$. birabeni foi capturada em todos os meses de coleta, com predominância no período seco $(n=50)$, no entanto, nos meses mais frios houve um decréscimo na abundância desses indivíduos $(\mathrm{n}=16)$. A espécie Corinna sp. também foi captura em quase todos os meses de coleta (exceto outubro), porém com maior predominância durante a estação chuvosa $(n=24)$ se comparada, por exemplo, aos meses mais quentes $(n=10)$ (Tabela 2).

De acordo com Jocqué (1988) e Sousa (2016), embora as atividades de forrageamento e reprodução de algumas espécies de aranhas estejam restritas ao período seco, outras espécies podem manter suas atividades normalmente em ambas as estações (seca e chuvosa), por exemplo, algumas espécies da família Corinnidae podem ser registradas tanto no período seco como chuvoso, com precipitação variando de média a alta.

\section{CONSIDERAÇÕES FINAIS}

Os resultados obtidos permitem inferir temporalmente e localmente, que a composição da fauna de aranhas do solo nos dois fragmentos da APA do Inhamum (Mata de Galeria e Cerrado censo stricto) difere entre si. As famílias Corinnidae e Oonopidae foram as que apresentaram maior riqueza de espécies e a família Zodariidae, com o maior número de indivíduos, sendo as espécies L. birabeni e Corinna sp. 1 as mais abundantes. O presente trabalho constitui o primeiro inventário da fauna de aranhas do solo na APA do Inhamum, Caxias, Maranhão.

Espera-se que este estudo possa incentivar novas pesquisas, principalmente nas áreas de preservação existentes no município, contribuindo, desse modo, para o conhecimento da araneofauna do solo local e para a preservação do meio ambiente. A preservação de fragmentos florestais, impactados ou não é importante para a conservação e a manutenção das espécies de aranhas do Bioma Cerrado, ainda bastante desconhecidas, especialmente na região Nordeste do Brasil

Tabela 1 - Composição das aranhas de solo coletadas em duas fitofisionomias de Cerrado na APA do Inhamum, Caxias - MA, no período de outubro/2017 a abril/2018. Fonte: elaborado pelos autores (2019)

\section{Fitofisionomias}

\begin{tabular}{|c|c|c|c|}
\hline Famílias/Espécies & Área $1(\%)$ & Área $2(\%)$ & Total \\
\hline \multicolumn{4}{|l|}{ Caponiidae* } \\
\hline Caponina sp. (Simon, 1892) NR/R & $1(0.90)$ & 0 & 1 \\
\hline \multicolumn{4}{|l|}{ Corinnidae } \\
\hline Abapeba sp. (Bonaldo, 2000) NR/D & $1(0.90)$ & $1(0.70)$ & 2 \\
\hline Attacobius sp. (Mello-Leitão, 1925) ${ }^{\mathrm{R}}$ & $1(0.90)$ & 0 & 1 \\
\hline Castianeira sp.1 (Keyserling, 1879) ${ }^{R}$ & $1(0.90)$ & 0 & 1 \\
\hline Castianeira sp.2 (Keyserling, 1879) ${ }^{\mathrm{D}}$ & $1(0.90)$ & $1(0.70)$ & 2 \\
\hline Corinna sp.1 (C. L. Koch, 1841) & $16(14.41)$ & $18(12.68)$ & 34 \\
\hline Corinna sp.2 (C. L. Koch, 1841) ${ }^{D}$ & $1(0.90)$ & $1(0.70)$ & 2 \\
\hline Parachemmis sp. (Chickering, 1937) NR & $3(2.70)$ & $1(0.70)$ & 4 \\
\hline \multicolumn{4}{|l|}{ Ctenidae } \\
\hline Ancylometes rufus (Walckenaer, 1837) NR/R & 0 & $1(0.70)$ & 1 \\
\hline Ctenus sp.1 (Walckenaer, 1805) ${ }^{\mathrm{NR}}$ & $8(7.21)$ & $4(2.82)$ & 12 \\
\hline Ctenus sp.2 (Walckenaer, 1805) NR/D & $2(1.80)$ & 0 & 2 \\
\hline
\end{tabular}




\section{Gnaphosidae}

Apopyllus sp. (Platnick \& Shadab, 1984)

Camillina sp. (Berland, 1919)

$1(0.90)$

$8(5.63)$

Hahniidae**

Neohahnia sp. (Mello-Leitão, 1917) NR/R

0

$1(0.70)$

1

\section{Idiopidae}

Idiops sp.1 (Perty, 1833) NR/R

0

$1(0.70)$

1

Idiops sp.2 (Perty, 1833) NR/R

$1(0.90)$

0

1

\section{Linyphiidae}

Meioneta sp. (Millidge, 1991) NR/R

Sphecozone sp. (O. Pickard-Cambridge, 1871) NR

$2(1.80)$

$1(0.70)$

\section{Miturgidae $^{* * *}$}

Teminius insularis (Lucas, 1857) ${ }^{\mathrm{D}}$

0

$2(1.41)$

Mysmenidae*

Microdipoena sp. (Banks, 1895) NR/R

$1(0.90)$

0

\section{Ochyroceratidae}

Ochyrocera sp. (Simon, 1892) ${ }^{\mathrm{NR}}$

$15(13.51)$

$1(0.70)$

\section{Oonopidae}

Gamasomorpha sp. (Karsch, 1881) NR/D

$1(0.90) \quad 1(0.70) \quad 2$

Hexapopha sp.1 (Platnick, Berniker \& Víquez, 2014) NR

3 (2.70)

$3(2.11)$

Hexapopha sp.2 (Platnick, Berniker \& Víquez, 2014) NR/R

$1(0.90)$

$$
0
$$

Oonopoides sp.1 (Bryant, 1940) NR

Oonopoides sp.2 (Bryant, 1940) NR/D

$2(1.80)$

0

2

\section{Palpimanidae}

Otiothops sp. (MacLeay, 1839) 


\section{Pholcidae}

Kambiwa sp. (Huber, 2000) ${ }^{\mathrm{NR}}$

$13(11.71)$

$11(7.75)$

Pisauridae*

Architis sp. (Simon, 1898) NR/R

$1(0.90)$

0

1

Prodidomidae**

Prodidomus sp. (Lucas, 1846) ${ }^{\mathrm{NR} / \mathrm{R}}$

0

$1(0.70)$

1

\section{Salticidae}

Asaphobelis sp. (Simon, 1902) NR/R

$1(0.90)$

0

1

Chira sp. (Peckham \& Peckham, 1896) NR/R

0

$1(0.70)$

1

Soesilarishius sp. (Makhan, 2007) NR

$2(1.80)$

$2(1.41)$

4

Sicariidae ${ }^{* *}$

Loxosceles amazonica (Gertsch, 1967) ${ }^{\mathrm{R}}$

0

$1(0.70)$

1

Scytodidae ${ }^{* *}$

Scytodes sp. (Latreille, 1804) NR/R

0

$1(0.70)$

1

Theraphosidae**

Hapalopus sp. (Ausserer, 1875) NR/R

0

$1(0.70)$

1

Theridiidae* $^{*}$

Latrodectus sp. (Walckenaer, 1805) NR/R

$1(0.90)$

0

1

Titanoecidae

Goeldia sp. (Keyserling, 1891) NR

$1(0.90)$

$3(2.11)$

4

Trachelidae

Orthobula sp. (Simon, 1897) NR/D

$1(0.90)$

$1(0.70)$

2

Zodariidae

Epicratinus sp. (Jocqué \& Baert, 2005) ${ }^{\mathrm{NR}}$

$1(0.90)$

$12(8.45)$

13

Leprolochus birabeni (Mello-Leitão, 1942)

$24(21.62)$

$42(29.58)$

66

TOTAL

$111(100 \%)$

$142(100 \%)$ 253 
Tabela 2 - Presença e ausência das aranhas de solo coletadas durante os períodos seco e chuvoso na APA do Inhamun, Caxias - MA, no período de outubro/2017 a abril/2018. Fonte: elaborado pelos autores (2019).

\begin{tabular}{|c|c|c|c|c|c|c|c|c|c|c|c|c|c|c|c|}
\hline & & & & & & & & \multirow{2}{*}{\multicolumn{7}{|c|}{ ÁREA 2}} & \multirow[b]{4}{*}{$\mathbf{T}$} \\
\hline \multirow{3}{*}{$\begin{array}{l}\text { Espécies/Meses } \\
\text { Thes }\end{array}$} & \multicolumn{7}{|c|}{ ÁREA 1} & & & & & & & & \\
\hline & \multicolumn{3}{|c|}{ Seco } & \multicolumn{4}{|c|}{ Chuvoso } & \multicolumn{3}{|c|}{ Seco } & \multicolumn{4}{|c|}{ Chuvoso } & \\
\hline & 10 & 11 & 12 & 01 & 02 & 03 & 04 & 10 & 11 & 12 & 01 & $\mathbf{0 2}$ & 03 & 04 & \\
\hline Abapeba sp. & - & - & - & - & - & 1 & - & - & - & - & - & - & 1 & - & 2 \\
\hline Ancylometes rufus & - & - & - & - & - & - & - & - & 1 & - & - & - & - & - & 1 \\
\hline Apopyllus sp. & - & - & 1 & - & - & - & - & - & - & - & - & 1 & 1 & - & 3 \\
\hline Architis sp. & 1 & - & - & - & - & - & - & - & - & - & - & - & - & - & 1 \\
\hline Asaphobelis sp. & - & - & - & - & - & - & 1 & - & - & - & - & - & - & - & 1 \\
\hline Attacobius sp. & - & - & - & - & - & - & 1 & - & - & - & - & - & - & - & 1 \\
\hline Camillina sp. & - & - & - & - & - & 1 & - & - & - & - & 1 & 7 & - & - & 9 \\
\hline Caponina sp. & - & - & - & - & 1 & - & - & - & - & - & - & - & - & - & 1 \\
\hline Castianeira sp.1 & 1 & - & - & - & - & - & - & - & - & - & - & - & - & - & 1 \\
\hline Castianeira sp.2 & - & - & - & 1 & - & - & - & - & - & - & 1 & - & - & - & 2 \\
\hline Chira sp. & - & - & - & - & - & - & - & - & - & - & - & 2 & - & - & 2 \\
\hline Corinna sp.1 & - & 3 & 6 & 2 & 1 & 2 & 2 & - & 1 & - & 11 & 6 & - & - & 34 \\
\hline Corinna sp. 2 & - & 1 & - & - & - & - & - & - & - & - & - & - & - & 1 & 2 \\
\hline Ctenus sp.1 & - & - & - & 3 & 5 & - & - & - & 2 & - & 1 & - & 1 & - & 12 \\
\hline Ctenus sp.2 & - & - & - & 1 & 1 & - & - & - & - & - & - & - & - & - & 2 \\
\hline Epicratinus sp. & - & - & 1 & - & - & - & - & - & - & - & 7 & 5 & - & - & 13 \\
\hline Gamasomorpha sp & - & - & 1 & - & - & - & - & 1 & - & - & - & - & - & - & 2 \\
\hline Goeldia sp. & 1 & - & - & - & - & - & - & 3 & - & - & - & - & - & - & 4 \\
\hline Hapalopus sp. & - & - & - & - & - & - & - & - & 1 & - & - & - & - & - & 1 \\
\hline Hexapopha sp.1 & - & - & - & 1 & - & 2 & - & - & - & - & - & 1 & 2 & - & 6 \\
\hline Hexapopha sp.2 & 1 & - & - & - & - & - & - & - & - & - & - & - & - & - & 1 \\
\hline Idiops sp.1 & - & - & - & - & - & - & - & - & - & - & 1 & - & - & - & 1 \\
\hline Idiops sp.2 & - & - & - & - & - & - & 1 & - & - & - & - & - & - & - & 1 \\
\hline
\end{tabular}




\begin{tabular}{|c|c|c|c|c|c|c|c|c|c|c|c|c|c|c|c|}
\hline Kambiwa sp. & 4 & 3 & 4 & 1 & - & 1 & - & 4 & 1 & 3 & 3 & - & - & - & 24 \\
\hline Latrodectus sp. & - & - & - & - & - & - & 1 & - & - & - & - & - & - & - & 1 \\
\hline Leprolochus birabeni & 11 & 6 & 5 & - & 1 & - & 1 & 17 & 7 & 4 & 4 & 3 & 5 & 2 & 66 \\
\hline Loxosceles amazonica & - & - & - & - & - & - & - & - & - & - & - & 1 & - & - & 1 \\
\hline Meioneta sp. & - & - & - & - & - & - & - & - & - & - & - & 1 & - & - & 1 \\
\hline Microdipoena sp. & - & - & - & - & - & - & 1 & - & - & - & - & - & - & - & 1 \\
\hline Neohahnia sp. & - & - & - & - & - & - & - & - & - & - & - & - & - & 1 & 1 \\
\hline Neotrops sp. & - & - & - & - & - & - & - & 1 & 1 & 2 & - & - & - & - & 4 \\
\hline Neoxyphinus sp. & - & - & - & - & - & - & - & - & 2 & 1 & - & - & 1 & - & 4 \\
\hline Ochyrocera sp. & - & - & 1 & 3 & 2 & 4 & 5 & - & - & - & - & - & - & 1 & 16 \\
\hline Oonopoides sp.1 & - & - & 1 & - & - & - & - & 1 & - & - & - & - & 7 & 1 & 10 \\
\hline Oonopoides sp.2 & - & - & 1 & 1 & - & - & - & - & - & - & - & - & - & - & 2 \\
\hline Orthobula sp. & - & 1 & - & - & - & - & - & 1 & - & - & - & - & - & - & 2 \\
\hline Otiothops sp. & 1 & 1 & - & - & - & - & - & - & - & - & - & - & 1 & - & 3 \\
\hline Parachemmis sp. & - & - & 1 & 1 & 1 & - & - & - & - & - & - & - & 1 & - & 4 \\
\hline Prodidomus sp. & - & - & - & - & - & - & - & - & 1 & - & - & - & - & - & 1 \\
\hline Scytodes sp. & - & - & - & - & - & - & - & - & - & 1 & - & - & - & - & 1 \\
\hline Soesilarishius sp. & - & 1 & - & - & - & 1 & - & - & - & - & - & 1 & 1 & - & 4 \\
\hline Sphecozone sp. & - & - & - & 1 & 1 & - & - & - & - & - & 1 & - & - & & 3 \\
\hline Teminius insularis & - & - & - & - & - & - & - & 1 & - & - & - & - & - & - & 1 \\
\hline TOTAL & & & & & & & & & & & & & & & 253 \\
\hline
\end{tabular}

$10=$ outubro $; \mathbf{1 1}=$ novembro $; \mathbf{1 2}=$ dezembro $; 01=$ janeiro $; 02=$ fevereiro $; 03=$ março $; 04=$ abril $; \mathbf{T}=$ total . 


\section{REFERÊNCIAS}

ALBUQUERQUE, A. Riacho Pontes e a Área de Proteção Ambiental Municipal do Inhamum, Caxias/MA. In: BARROS, M. C. et al. (Org.). Biodiversidade na Área de Proteção Ambiental do Inhamum. São Luís: UEMA, 2012. p. 22-25.

AZEVEDO, R. et al. Aranhas epígeas de um fragmento de mata em área urbana em Fortaleza, Ceará, Brasil. Research and Teaching in Exact and Natural Sciences, v. 1, n. 2, p. 104114, 2017.

BRESCOVIT, A. D.; OLIVEIRA, U.; SANTOS, A. J. Aranhas (Araneae, Arachnida) do Estado de São Paulo, Brasil: diversidade, esforço amostral e estado do conhecimento. Biota Neotropica, v. 11, n. 1, p. 717-747, 2011.

CARVAlHO, L. S.; AVElinO, M. T. L. Composição e diversidade da fauna de aranhas (Arachnida, Araneae) da Fazenda Nazareth, Município de José de Freitas, Piauí, Brasil. Biota Neotropica, v. 10, n. 3, p. 1-11, 2010.

CONCEIÇÃO, G. M.; CASTRO, A. A. J. F. Fitossociologia de uma área de cerrado marginal, Parque Estadual do Mirador, Mirador, Maranhão. Scientia Plena, v. 5, n. 10, p. 1-16, 2009.

CONCEIÇÃO, G. M.; da SILVA, D. S.; RODRIGUES, M. S. Aspectos florísticos e ecológicos da família convolvulaceae da área de proteção ambiental municipal do Inhamum, Caxias, Maranhão, Brasil. Brazilian Geographical Journal: Geosciences and Humanities research medium, v. 5, n. 2, p. 595-613, 2014.

CUNHA, J. A. S. et al. Diversidade preliminar de aranhas de solo em áreas de Cerrado Litorâneo com diferentes níveis de conservação, Maranhão, Brasil. Revista Biociências, v. 18, n. 1, p. 5-13, 2012.

da SILVA, G. S. et al. Trilhas ecológicas da área de proteção ambiental municipal do Inhamum e seus aspectos florísticos, maranhão, Brasil. Agrarian Academy, v. 3, n. 6, p. 87, 2016.

DIAS, M. F. R.; BRESCOVIT, A. D.; MENEZES, M. Aranhas de solo (Arachnida: Araneae) em diferentes fragmentos florestais no sul da Bahia, Brasil. Biota Neotropica, v. 5, n. 1, p. 1-10, 2005.

DRESCHER, M. S.; ELTZ, F. L. F.; ROVEDDER, A. P. M.; DORNELES F. O. Mesofauna como bioindicador para avaliar a eficiência da revegetação com Lupinus albescens em solo arenizado do sudoeste do Rio Grande do Sul. In: XXXI Congresso Brasileiro de Ciência do Solo, Gramado, SBCS. 2007.

FERRO, C. E. Diversidade de aranhas (Araneae) de solo de uma área de mata ciliar, junto ao rio Ibicuí-Mirim, em Itaara, Rio Grande do Sul, Brasil. Dissertação de Mestrado apresentado ao Programa de Pós-Graduação em Ecologia e
Evolução da Biodiversidade da Universidade Pontifícia Católica do Rio Grande do Sul. 2008. 82 p.

FOELIX, R. F. Biology of Spiders. Oxford University Press, Oxford, 2011. 419 p.

FORNAZIER, R.; GATIBONI, L. C.; WILDNER, L. P.; BIANZI, D.; TODERO, C. Modificações na fauna edáfica durante a decomposição da fitomassa de Crotalaria juncea L. In: XXXI Congresso Brasileiro de Ciência do Solo, Gramado, SBCS. 2007.

FRANCISCO, R. C. Estudo da comunidade de aranhas (Araneae: Arachnida) de solo como ferramenta de diagnóstico ambiental. Porto Alegre, 2011. 75 f. Dissertação (Mestrado em Zoologia). Programa de Pós-Graduação em Zoologia, Universidade Pontifícia Católica do Rio Grande do Sul, Porto Alegre - RS, 2011.

GIRACCA, E. M. N. et al. Levantamento da meso e macrofauna do solo na microbacia do Arroio Lino, Agudo, RS. Revista Brasileira de Engenharia Agrícola e Ambiental, v. 9, n. 3, p. 257-261, 2003.

JOCQUÉ, R. An updating of the genus Leprolochus (Araneae: Zodariidae). Studies on Neotropical Fauna and Environment, v. 23, n. 2, p. 77-87, 1988

LEITE, L. F. C.; FREITAS, R. C. A.; SAGRILO, E.; GALVÃO, S. R. S. Decomposição e liberação de nutrientes de resíduos vegetais depositados sobre Latossolo Amarelo no Cerrado Maranhense. Revista Ciência Agronômica, v. 41, n. 1, p. 2935,2010 .

LIMA, J. S. et al. Diversidade de aranhas em área remanescente de Mata Atlântica, na vila Bananeira, zona rural de Arapiraca, agreste Alagoano. Revista Ambientale, v. 11, n. 2, p. 1-12, 2019.

MORAES, R. C. B. et al. Software para análise faunística ANAFAU. In: Simpósio de Controle Biológico, 8, São Pedro, SP. Piracicaba: ESALQ/USP, 2003. p. 195.

MORAES, V. S. Efeitos da estrutura da vegetação na composição da assembleia de aranhas (Arachnida: Araneae) em estrato arbóreo de diferentes fitofisionomias do Cerrado. Brasília, 2014. 61 f. Dissertação (Mestrado em Ecologia). Programa de Pós-Graduação em Ecologia, Universidade de Brasília, Brasília - DF, 2014.

NERES, L. P.; CONCEIÇÃO, G. M. Florística e fitossociologia da área de proteção ambiental municipal do Inhamum, Caxias, Maranhão, Brasil. Cadernos de Geociências, v. 7, n. 2, p. 122-130, 2010.

OLIVEIRA, U.; BRESCOVIT, A. D.; SANTOS, A. J. Sampling effort and species richness assessment: a case study on Brazilian spiders. Biodiversity and Conservation, v. 26, n. 6, p. 1481-1493, 2017. 
ROCHA, L. M. Fauna de aranhas edáficas (Arachnida: Araneae) em um fragmento periurbano em ambiente de Mata Atlântica no Sul do Brasil. Criciúma, 2017. 28 f. Trabalho de Conclusão de Curso (Bacharel em Ciências Biológicas). Curso de Bacharel em Ciências Biológicas, Universidade do Extremo Sul Catarinense - UNESC, Criciúma - SC, 2017.

SANTANA, R. C. Community structure and composition of litter spiders (Arachnida: Araneae) and influence of macroclimatic factors on Parque Ecológico Jatobá Centenário, Morrinhos, Goiás, Brazil. Journal of Threatened Taxa, v. 7, n. 10, p. 7612-7624, 2015.

SILVA, G. S. et al. Trilhas ecológicas da área de proteção ambiental municipal do Inhamum e seus aspectos florísticos, Maranhão, Brasil. Revista Agrária Acadêmica, v. 3, n. 6, p. 86-98, 2016.

SOUSA, J. A. Comunidade de artrópodes de serapilheira em uma área de Cerrado do Nordeste do estado do Maranhão, Brasil. Chapadinha, 2016. 39 f. Trabalho de Conclusão de Curso (Licenciatura e Bacharel em Ciências Biológicas).
Curso de Licenciatura e Bacharel em Ciências Biológicas, Universidade Federal do Maranhão, Chapadinha - MA, 2016.

WORLD SPIDER CATALOG (2020). World Spider Catalog. Version 21.0. Natural History Museum Bern, online at http://wsc.nmbe.ch, accessed on May 16, 2020. Doi: $10.24436 / 2$

\section{AGRADECIMENTOS}

Agradecemos aos colaboradores do Laboratório de Fauna do Solo-LAFS, da Universidade Estadual do Maranhão, na realização das coletas e a todos os funcionários do Instituto Butantan pela ajuda na identificação das aranhas, especialmente aos integrantes do Laboratório de Coleções Zoológicas (LECZ) sob a coordenação do Prof. Dr. Antônio Domingos Brescovit (Bolsista CNPq, processo 303903/20019-8).

Recebido em: 16/10/2019

Aceito para publicação em: 24/06/2020 